\title{
EFEITO DE CULTIVARES DE ALGODOEIRO SOBRE A BIOLOGIA E CAPACIDADE PREDATÓRIA DE ORIUS INSIDIOSUS (SAY, 1832) (HEMIPTERA: ANTHOCORIDAE) PREDANDO APHIS GOSSYPII GLOVER, 1877 (HEMIPTERA: APHIDIDAE)
}

\section{J.E.M. Oliveira ${ }^{1}$, S.A. De Bortoli ${ }^{1}$, R.F. dos Santos $^{1}$, L.C.P. Silveira ${ }^{2}$}

${ }^{1}$ Universidade Estadual Paulista, Faculdade deCiências Agráriase Veterinárias, Departamento deFitossanidade, Laboratório de Biologia e Criação de Insetos, Via de Acesso Prof. Paulo Donato Castellane s/no, CEP14884-900, Jaboticabal, SP, Brasil. E-mail: eudes@fcav.unesp.br

\section{RESUMO}

Estudou-se a sobrevivência, a reprodução e a capacidade predatória de Orius insidiosus (Say) sobre o pulgão do algodoeiro A phis gossypii Glover. Plantas de algodoeiro das cultivares Antares, CNPA7H e Acala 90 (respectivamente, glabra, de média pilosidade e pilosa) foram individualizadas e infestadas com quinze ninfas de terceiro/quarto estádio de A. gossypii e, em seguida, liberadas ninfas do predador O.insidiosus a partir do primeiro estádio ninfal. As avaliações foram realizadas diariamente, quantificando-se sobrevivência e desenvolvimento ninfal; o número de pulgões predados por dia e total; o número de ovos e a população do predador (total de indivíduos gerados pelas fêmeas) e determinada a eficiência de conversão alimentar através da taxa de predação de $O$. insidiosus. O desenvolvimento ninfal não foi afetado pelo cultivar de algodoeiro. Ocultivar Antares favoreceu maior taxa diária de predação para os dois primeiros e quarto estádio ninfal e fase adulta de $O$. insidiosus. O número de ovos e de ninfas foi menor quando as fêmeas de O. insidiosus foram confinadas no cultivar Acala 90. A eficiência de conversão alimentar nos cultivares Antares, CNPA7He Acala 90 foram, respectivamente, 4,28;3,00 e 2,75 pulgões predados para cada ovo gerado pelo predador.

PALAVRAS-CHAVE: Controle biológico, Orius insidiosus, Aphis gossypii, resistência de plantas, algodão.

\section{ABSTRACT}

EFFECTOF COTTON CULTIVARSON BIOLOGICAL ASPECTS ANDPREDATIONBEHAVIOR OF ORIUS INSIDIOSUS (SAY) PREYING ON APHIS GOSSYPII GLOVER. The objective of this research was to determine the survival, reproduction and predaceous capacity of Orius insidiosus on cotton aphid Aphis gossypii. Cotton plants of Antares, CNPA7H and Acala 90 cultivars (respectively, without tricome, medium tricome density and high tricome density) were individualized and infested with 15 third/fourth instar nymphs of $A$. gossypii, and then first-instar nymphs of O.insidiosus were released on the plants. The evaluations were made daily, quantifying survival and nymphal development; the number of cotton aphids $A$. gossypii per day and total; the number of eggs and the population of the predator (number of insects by female); and the conversion efficiency of $A$. gossypii predaceous. The nymphal development did not differ according to the cotton cultivars. The Antares cultivar favored a higher daily predation rate for the 1 st, 2 nd and 4 th instars and adults of $O$. insidiosus. The number of eggs and nymphs was smaller when O. insidiosus females were confined on the Acala 90 cultivar. According to the predation rate of $O$. insidiosus, the efficiency of alimentary conversion was determined for the Antares, CNPA7H and Acala 90 cultivars, being respectively, 4.28, 3.00 and 2.75 cotton aphid predation for each egg of the predator.

KEY WORDS: Biological control, minute pirate bug, plant resistance, predation behavior.

\section{INTRODUÇAO}

O algodoeiro pode sofrer dano em todas as suas fases de desenvolvimento por diversos insetos, com danos significativos e perda de produção. A presença de espécies pragas, particularmente afídeos, tripes, mirídeos, lagartas, percevejos e ácaros, é comum em todos os sistemas de produção de algodão do mundo.

\footnotetext{
${ }^{2}$ Universidade Federal de Lavras, Departamento de Entomologia, Lavras, MG, Brasil.
} 
A maioria destas pragas é polífaga, sendo a produção do algodoeiro influenciada, portanto, pela comunidade de artrópodes presentes (LutTRell et al., 1994).

A espécie de pulgão, Aphis gossypii Glover, 1877 (Hemiptera: Aphididae), tem sido constantemente relatada na cultura do algodoeiro, com infestações freqüentes e registro de ocorrência principalmente no início da cultura (CAUQuil, 1981; DenÉCHÈre, 1981). Essa espécie de pulgão é considerada como sendo a mais comum na cultura do algodoeiro, causando danos diretos através da sucção contínua da seiva (BRIOso, 1996), e indiretos pela transmissão de viroses (Peña-Martínez, 1992).

Para ouso racional doagroecossistema cotonícola, é importante que se utilize todos os métodos disponíveis para o controle das pragas, dando preferência sempreà utilização e preservaçãono agroecossistema dos controladores biológicos, os quais podem ser associados com o método de resistência de plantas a insetos.

A família Anthocoridae é constituída por pequenos insetos e possui cerca de 600 espécies, ocupando diversos habitats, desde vegetação nativa até diferentes agroecossistemas (LATTIN, 1999; LatTin, 2000). No Brasil, a espécie Oriusinsidiosus (Say, 1832) (Hemiptera: Anthocoridae) é considerada como a mais abundante e de maior potencial para utilização em programas de controle biológico de diversas pragas (BUENO, 2000).

$O$. insidiosus possui certas características que o torna promissor agente de controle biológico, destacando-se a alta eficiência de busca, habilidade para aumentar a população e agregação rápida quando há presas em abundância, além de sobreviver em baixa densidade de presas (Bushet al., 1993). Esse predador é considerado uma espécie generalista, possuindo habilidade de se alimentar de diferentes presas e substratos e de se abrigar em diferentes agroecossistemas, o que o torna apto à exploração do ecossistema e a sobreviver naturalmente (SILVEIRA et al., 2003).

O tipo de alimento pode interferir em vários parâmetros biológicos de $\mathrm{O}$. insidiosus, como sobrevivência, longevidade, fecundidade e viabilidade dos ovos, podendo inclusive levá-lo a não completar o desenvolvimento (RICHARDS; SCHMIDT, 1996a; BUENO, 2000; Mendes; Bueno, 2001). A fecundidade de $O$. insidiosus é diretamente afetada pelo alimento, sendo esse um fator de influência direta para o aumento rápido de sua população (KIMAN; YEARGAN, 1985; RICHARDS; SCHMIDT, 1996a). As fêmeas de O. insidiosus ovipositam endofiticamente em uma grande variedade de substratos naturais, como vagens de leguminosas, caules de feijão, brotos de batata, inflorescências de picão Bidens pilosa L. (Asteraceae), pecíolos de folhas de algodoeiro, folhas de gerânio, pepino, batateira, entre outros (BUENO, 2000). O substrato vegetal para oviposição pode afetar diretamente a fertilidade deO.insidiosus, sendo que uma das condições básicas exigidas é que o mesmo seja aceitável pelo predador (RICHARDS; SCHMIDT, 1996b).

Em relação ao comportamento de O. insidiosus, os adultos são considerados bons voadores e, como as ninfas, movimentam-se rapidamente na planta a procura de suas presas. Possuem um eficiente comportamento de busca, sendo capazes de agregarem-se em áreas de grande densidade da presa, condição essa mais freqüente para as ninfas do que para os adultos (BuENO, 2000).

A predação é um processo complexo, afetado por fatores básicos, como densidades da presa e do predador, e por fatores secundários, envolvendo as características do ambiente, da presa edo predador(HoLLING , 1961). A presença de predadores em um determinado ambiente e o seu efeito sobre a dinâmica da presa depende da habilidade do predador em encontrá-la, da sua densidade e qualidade (COHEN, 1998), ou seja, se houver presa em maior número e de melhor qualidade, certamente o predador mostrará boa resposta.

No Brasil, estudossobre bioecologia, quantificação e participação de O.insidiosus em agroecossistema do algodoeiro, tendo como presa o pulgão A. gossypii, ainda são escassos. Portanto, justifica-se a realização de estudos, a respeito da biologia e capacidade de predação desse percevejo sobre a presa A. gossypii.

\section{MATERIAL E MÉTODOS}

O experimento foi conduzido no Laboratório de Biologia e Criação de Insetos do Departamento de Fitossanidade da Faculdade de Ciências Agrárias e Veterinárias da Universidade Estadual Paulista FCAV/UNESP, Jaboticabal, São Paulo. As condições experimentais foram de $25 \pm 1^{\circ} \mathrm{C}, 70 \pm 10 \%$ deumidade relativa e $14 \mathrm{~h}$ de fotofase.

Plantio de algodoeiro. Plantas de algodoeiro (Gossypium hirsutum L. raçaLatifolium Hutch), Antares, CNPA 7He Acala 90 foram cultivadas em bandejas de isopor e mantidas em telados livres de pragas e inimigos naturais. Foram realizados plantios escalonados com intervalos de 10 dias para obtenção de folhas e plantas adequadas para a manutenção da criação do pulgão $A$. gossypii e condução do experimento.

Criação de Orius insidiosus. A criação do predador $O$. insidiosus foi iniciada a partir de espécimes coletados em agroecossitemas de algodão e milho no Campus da FCAV/UNESP, Jaboticabal, SP. A criação foi mantida em câmara climatizada tipo B.O.D. ajustada a $25 \pm 1^{\circ} \mathrm{C}$ e $70 \pm 10 \%$ de umidade relativa e $14 \mathrm{~h}$ de fotofase, adotando-se a metodologia adaptada de ISENHOUR; YEARGAN (1981), SCHMIDT et al. (1995), BUENO (2000) e Silveira; Bueno (2003). 

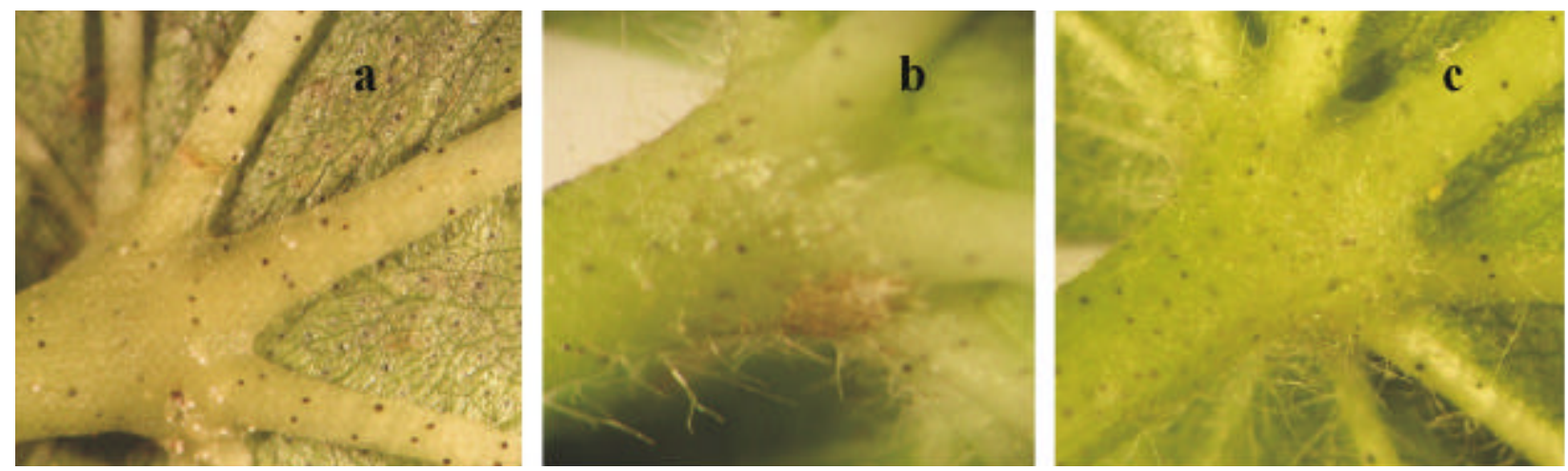

Fig. 1 - Presença de tricomas nos cultivares Antares (a), CNPAH7h (b) e Acala 90 (c). $25 \pm 1^{\circ} \mathrm{C}, 70 \pm 10 \%$ de umidade relativa e 14 h de fotofase.

Os adultos de O. insidiosus foram mantidos em gaiolas cilíndricas $(1,7 \mathrm{~L})$ vedadas com filme de PVC. No interior da gaiola colocou-se papel toalha, servindo como abrigo para os predadores. Para promover aeração foram feitos furos no filme de PVC, com auxílio de um estilete. Em cada recipiente de criação foram mantidos 50 casais do percevejo predador, ovos de Anagastakuehniella (Zeller, 1879) (Lepidoptera: Pyralidae), como fonte alimentar e inflorescências de B. pilosa, como substrato de oviposição, sendo estas últimas tratadas em solução de hipoclorito a $2 \%$ por cerca de 2 minutos que, após secagem, foram fixadas a um chumaço de algodão embebido em água destilada para a manutenção da turgescência e fornecimento da umidade aos predadores. As inflorescências contendoovos do predadorforam removidasetransferidas para placas de Petri $(14 \times 2 \mathrm{~cm})$.

Um dia antes do previsto para eclosão das ninfas, ovos de A. kuehniella foram colocados no topo da inflorescência como fonte de alimento para as formas jovens. As ninfas do predador ao eclodirem permaneceram no interior da mesma placa por todo período ninfal. No interior da placa foi colocado papel toalha, servindo como abrigo para os predadores. Para promover aeração foram feitos furos no filme dePVC, com auxílio de um estilete. Em cada recipiente de criação das ninfas foram mantidos cerca de 100 indivíduos, sendo o fornecimento de água feito por um chumaço de algodão embebido com água destilada. Oalimento ea água foram renovados a cada dois dias.Os adultos, logo após a emergência, foram separados em casais para iniciar o novo ciclo da criação.

Criação Aphis gossypii. Os pulgões utilizados nos experimentos foram oriundos da criação mantida no laboratórioem plantas docultivarIAC24.Essa criaçãofoi iniciadacomindivíduoscoletadosemplantas dealgodoeiro de plantios comerciais da região de Jaboticabal, São Paulo, sendo posteriormente transferidos para plantas, mantidas em telado sob condições de laboratório, protegidas, para evitar a migração e infestação de outras espécies de pulgões e de inimigos naturais. Periodica- mente, as colônias dos pulgões foram transferidas para novas plantas, com 20 dias de emergência (OliveIRAetal., 2005; De Bortoli; Oliveira, 2005).

A caracterização das cultivares de algodoeiro foi feita quanto à presença e número de tricomas. Os testes foram conduzidos utilizando-se as cultivares Antares, CNPA7He Acala 90, respectivamente, glabra, de pilosidade média e pilosa (Fig. 1) verificando-se a sua influência sobre os aspectos biológicos e a capacidade predatória de $O$. insidiosus sobre ninfas de terceiro e quarto estádios de $A$. gossypii.

Fase jovem. $\mathrm{O}$ experimento foi iniciado com 75 ninfas de primeiro instar, oriundas de criação mantida emlaboratório. Asninfasforamagrupadasemcinco por gaiola (placas de Petri - $14 \times 2 \mathrm{~cm}$ ) contendo no seu interiorumafolhadealgodoeirodecadacultivar, inserida num tubo com água (tipo anestésico odontológico) com um chumaço de algodão. Diariamente foram disponibilizados em cada tratamento/cultivar, 15 pulgões de terceiro/quarto estádio e, a cada 24 h da oferta, os insetos, predados ou não, foram repostos ou substituídos por indivíduos de idade semelhante ao descrito. As avaliações foram realizadas a cada $24 \mathrm{~h}$, observandosea duração, sobrevivência ecapacidade predatória em cada instar e total da fase ninfal.

Fase adulta. Ao atingirem a fase adulta, as fêmeas de $O$. insidiosus provenientes das ninfas mantidas nos seus respectivos tratamentos, foram acasaladas, mantendo-se os machos durante três dias (período suficiente para efetivação da cópula). Fêmea deO.insidiosus foi individualizada (placas de Petri $-14 \times 2 \mathrm{~cm}$ ) contendo no seu interior uma folha de algodoeiro de cada cultivar, inserida num tubo com água (tipo anestésico odontológico) com um chumaço de algodão. Diariamente foram disponibilizados em cada tratamento/cultivar, quinze pulgões deterceiro/quarto estádioe, a cada $24 \mathrm{~h}$ da oferta, os insetos, predados ou não, foram repostos ou substituídos por indivíduos de idade semelhante ao descrito. Avaliou-se a longevidade; períodos de pré-oviposição, reprodução, pós-oviposição e incubação; número total de 
ovos e deninfas por fêmea; taxa diária de produção de ovos e ninfas, em função do período reprodutivo; viabilidade dos ovos e o consumo médio diário e total da presa $A$. gossypii, além da eficiência de conversão alimentar $[\mathrm{ER}=$ número total de ovos/número total de pulgões predados], determinado a partir da taxa de predação por fêmeas do predador.

Os dados de duração e sobrevivência de ninfas, longevidade e reprodução de fêmeas, e capacidade predatória nas duas fases de desenvolvimento do predador, foram submetidos à ANOVA simples, e as médias comparadas pelo teste de Tukey a 5\% de probabilidade.

\section{RESULTADOS E DICUSSÃO}

O desenvolvimento (dias) de todos os estádios e na fase ninfal não foi afetado pelo cultivar de algodoeiro (Tabela1), apesar das ninfas mantidas nos cultivares com presença de tricomas apresentarem um alongamento em todos os estádios e no período ninfal. Período semelhante de desenvolvimento na fase ninfal (11,2 dias) foi observado por MENDEs et al.
(2003), quando as ninfas de O. insidiosus tiveram disponiblidade diária de ninfas de A. gossypii no $1^{\circ}$, $2^{\circ}$ e $3^{\circ}$ estádios ninfais. TommAsini et al. (2004) observaram semelhança no período de desenvolvimento de $O$. insidiosus alimentados com adultos de Frankliniella occidentalis (Pergande, 1895) para os dois primeiros e último estádios ninfa is $(2,1 ; 1,5$ e 3,4 dias, respectivamente) e para toda fase ninfal (10 dias). Mendes; Bueno (2001) relataram que ninfas de O. insidiosus apresentam variações nos estádios ninfais de 1,9 a 3,1 dias, para o primeiro e quinto estádios ninfais, respectivamente, quando alimentadas com Caliothrips phaseoli (Hood, 1912). KIMAN; YEARGAN (1985) observaram que, o tempo de desenvolvimento de $O$. insidiosus foi de 15,8 dias quando alimentado com Sericothrips variabilis (Beach, 1896). Esses autores ainda relataram que, quando a presa era ovos de Heliothis virescens (Fabr., 1781), o tempo de desenvolvimento foi de até 13,4 dias. MCCAFFREY; Horsburgh (1986) relataram que, quando alimentados com Panonychusulmi (Koch, 1836) e mantidos em temperatura de 23 e $29^{\circ} \mathrm{C}, \mathrm{O}$. insidiosus apresentou o tempo de desenvolvimento de 18,8 e 9,5 dias, respectivamente.

Tabela 1 - Duração média e sobrevivência de ninfas e da fase ninfal de Orius insidiosus predando Aphis gossypii em diferentes cultivares de algodoeiro. Temp. $25 \pm 1^{\circ}$ C; UR $70 \pm 10 \%$; fotofase 14h, Jaboticabal, 2006.

Instares

Tratamentos

\begin{tabular}{|c|c|c|c|c|c|c|}
\hline & \multicolumn{3}{|c|}{ Duração (dias) $^{1}$} & \multicolumn{3}{|c|}{ Sobrevivência (\%) } \\
\hline & Antares & CNPA7H & Acala 90 & Antares & CNPA7H & Acala 90 \\
\hline I & $2,17 \pm 0,38^{\mathrm{a}}$ & $2,38 \pm 0,49 a$ & $2,42 \pm 0,50 \mathrm{a}$ & 88,00 & 86,67 & 85,33 \\
\hline II & $1,52 \pm 0,50^{\mathrm{a}}$ & $1,59 \pm 0,50 a$ & $1,65 \pm 0,48 a$ & 81,33 & 78,67 & 76,00 \\
\hline III & $1,84 \pm 0,45^{\mathrm{a}}$ & $1,83 \pm 0,38 \mathrm{a}$ & $1,87 \pm 0,34 \mathrm{a}$ & 77,33 & 70,67 & 69,33 \\
\hline IV & $1,60 \pm 0,49^{a}$ & $1,66 \pm 0,48 a$ & $1,73 \pm 0,45 a$ & 66,67 & 66,67 & 65,33 \\
\hline V & $3,49 \pm 0,51^{\mathrm{a}}$ & $3,70 \pm 0,47 a$ & $3,85 \pm 0,36 a$ & 65,33 & 61,33 & 62,67 \\
\hline Fase ninfal & $10,59 \pm 0,70^{\mathrm{a}}$ & $11,20 \pm 0,58 a$ & $11,53 \pm 0,65 a$ & 60,00 & 56,00 & 57,33 \\
\hline
\end{tabular}

${ }^{1}$ Médias ( $\left.\pm \mathrm{DP}\right)$ seguidas de mesma letra, na mesma linha, não diferem entre si, pelo teste de Tukey $(\mathrm{P}>0,05)$.

Tabela 2 - Predação total e diária de ninfas e da fase ninfal deOrius insidiosus sobre Aphis gossypii, em diferentes cultivares de algodoeiro. Temp. $25 \pm 1^{\circ}$ C; UR $70 \pm 10 \%$; fotofase 14h, Jaboticabal, 2006.

\begin{tabular}{|c|c|c|c|c|c|c|}
\hline \multirow[t]{3}{*}{ Instares } & \multicolumn{6}{|c|}{ Tratamentos } \\
\hline & \multicolumn{3}{|c|}{ Total $^{1}$} & \multicolumn{3}{|c|}{ Diária $^{1}$} \\
\hline & Antares & CNPA7H & Acala 90 & Antares & CNPA7H & Acala 90 \\
\hline $\mathrm{I}$ & $7,86 \pm 1,64 a$ & $7,29 \pm 1,64 a$ & $7,31 \pm 1,50 \mathrm{a}$ & $3,64 \pm 0,50 a$ & $3,07 \pm 0,37 b$ & $3,04 \pm 0,34 a$ \\
\hline II & $7,26 \pm 2,48 a$ & $6,59 \pm 2,48 a$ & $6,74 \pm 2,14 a$ & $4,82 \pm 0,73 a$ & $4,09 \pm 0,68 b$ & $4,10 \pm 0,56 a$ \\
\hline III & $13,40 \pm 3,48 a$ & $13,04 \pm 3,08 a$ & $13,19 \pm 2,79 a$ & $7,28 \pm 0,88 a$ & $7,09 \pm 0,65^{a}$ & $7,07 \pm 0,66 a$ \\
\hline IV & $14,50 \pm 5,12 a$ & $14,42 \pm 4,52 \mathrm{a}$ & $13,00 \pm 3,81 a$ & $8,92 \pm 0,86 a$ & $8,68 \pm 0,80^{a}$ & $7,43 \pm 0,68 a$ \\
\hline $\mathrm{V}$ & $32,27 \pm 5,25 a$ & $32,80 \pm 4,19 a$ & $33,23 \pm 3,64 a$ & $9,25 \pm 0,84 a$ & $8,89 \pm 0,64^{a}$ & $8,63 \pm 0,46 a$ \\
\hline Fase ninfal & $75,73 \pm 7,48 a$ & $73,83 \pm 6,19 a$ & $73,57 \pm 6,24 a$ & $7,17 \pm 0,75 a$ & $6,60 \pm 0,46 a$ & $6,39 \pm 0,60 a$ \\
\hline
\end{tabular}

${ }^{1}$ Médias ( $\left.\pm \mathrm{DP}\right)$ seguidas de mesma letra, na mesma linha, não diferem entre si, pelo teste de Tukey $(\mathrm{P}>0,05)$. 
A sobrevivência ninfal de O. insidiosus diminui à medida que se avançam os estádios ninfais (Tabela 1). Os valores obtidos para esse parâmetro biológico de O. insidiosos estão deacordo comaqueles encontrados por Bushet al. (1993). No entanto, MENDEs et al. (2003) sugeriram que, com aumento da disponilibilidade de ninfas de $A$. gossypii, o predador responde positivamente a esse incremento, aumentando sua viabilidade ninfal. Tommasiniet al. (2004) observaram, para esse mesmo predador quando alimentado com adultos de F. occidentalis, uma sobrevivência da fase ninfal de 53,5\%. Mendes; Bueno (2001) relataram que para essa fase a sobrevivência das ninfas de $O$. insidiosus alimentadas C. phaseoli foi de $69 \%$, com mortalidade concentrada no $2^{\circ}$ estádio. Os autores ainda observaram que no $4^{\circ}$ e $5^{\circ}$ estádios não houve mortalidade ninfal.

Em relação à capacidade predatória, o estudo mostrou uma forte influência do cultivar de algodoeiro, indicando que ninfas do predador $O$. insidiosus mantidas em Antares tiveram maior taxa diária de predação para o $1^{\circ}, 2^{\circ}$ e $4^{\circ}$ estádios ninfais (Tabela 2). Independente do cultivar, a taxa diária de predação de O. insidiosus variou de 3,04 a 9,25 pulgões/dia do primeiro ao quinto ínstar do predador, enquanto que para toda a fase ninfal $O$. insidiosus predou até 75,73 ninfas de A. gossypii (Tabela 2). TommAsini et al. (2004) observaram que, na fase ninfal, O. insidiosus predou em média 39,2 adultos de F. occidentalis, com média diária de 3,9 insetos/ninfa. MENDES; BuENo (2001) relataram que, durante a fase ninfal, O. insidiosus apresentou uma taxa de predação variando de 6,1 a 29,3 adultos de $C$. phaseoli do primeiro ao quinto estádio ninfal, com consumo diário de 3,0 a 9,4 insetos, e em média 39,2 adultos de F.occidentalis, com predação média diária de
3,9 insetos/ninfa. As ninfas de O. insidiosus apresentam como comportamento a forma ativa e rápida de caminhamento pelo substrato a procura de abrigo e alimento (MENDEs; BuENO, 2001). Dessa forma, as ninfas de O. insidiosus podem ter sido inibidas, principalmente nos dois estádios iniciais de desenvolvimento, a percorrer de forma mais ativa a folha do algodoeiro, devidoà presença de tricomas nos cultivares $\mathrm{CNPA7H}$ e Acala 90. Mendesetal. (2003) observaram queem todos os estádios ninfais de $O$. insidiosus existe relação direta entre aumento de disponiblidade de presas (ninfas de A. gossypii) e consumo, além de que essa relação positiva pode interferir nos parâmetros de desenvolvimento desse predador. TOMMAsini et al. (2004) relataram haver um incremento significativo na relação ínstar e capacidade predatória de O. insidiosus, mostrando que independente da presa, à medida que os estádios avançam, a taxa de predação das ninfas pode duplicar entre estádios.

Fêmeas de $O$. insidiosus apresentaram maior número de ninfas e taxa de oviposição diária em toda fase no cultivar Antares, enquanto que o cultivar Acala 90 afetou negativamente o número de ovos e ninfas (Tabela3). Diariamente, as fêmeas do predador ovipositam mais ovos e num período mais curto (período de pré-oviposição), iniciando sua oviposição a partir de 2,69 dias, quando mantidas no cultivar Antares (Fig. 3). No entanto, quando essas fêmeas foram mantidas nos cultivares CNPA7H e Acala 90, esse período tendeu a se alongar (Tabela 3).

A longevidade, os períodos reprodutivo e de pósreprodução, deincubação deovos, o número deninfas / dia e a viabilidade de ovos não apresentaram diferença estatística significativa nos três cultivares testados (Tabela 3).

Tabela 3 - Características biológicas de fêmeas de Orius insidiosus predando Aphis gossypii em diferentes cultivares de algodoeiro. Temp. $25 \pm 1^{\circ}$ C; UR $70 \pm 10 \%$; fotofase 14h, Jaboticabal, 2006.

\begin{tabular}{lccc}
\hline Características & \multicolumn{3}{c}{ Tratamentos $^{1}$} \\
\cline { 2 - 4 } & Antares & CNPA7H & Acala 90 \\
\hline Longevidade de fêmeas (dias) & $26,23 \pm 13,01 \mathrm{a}$ & $21,80 \pm 6,06 \mathrm{a}$ & $20,73 \pm 6,45 \mathrm{a}$ \\
Período de pré-oviposição (dias) & $2,69 \pm 0,75 \mathrm{a}$ & $3,90 \pm 0,87 \mathrm{~b}$ & $3,73 \pm 0,65 \mathrm{~b}$ \\
Período reprodutivo (dias) & $20,76 \pm 9,13 \mathrm{a}$ & $16,40 \pm 5,31 \mathrm{a}$ & $15,64 \pm 5,71 \mathrm{a}$ \\
Período de pós-oviposição (dias) & $2,77 \pm 4,18 \mathrm{a}$ & $1,50 \pm 0,84 \mathrm{a}$ & $1,36 \pm 0,67 \mathrm{a}$ \\
Período de incubação (dias) & $3,96 \pm 0,47 \mathrm{a}$ & $3,85 \pm 0,70 \mathrm{a}$ & $3,73 \pm 0,75 \mathrm{a}$ \\
Número de ovos/fêmea & $33,07 \pm 14,45 \mathrm{a}$ & $23,30 \pm 7,43 \mathrm{ab}$ & $22,27 \pm 7,52 \mathrm{~b}$ \\
Número de ninfas/fêmea & $23,53 \pm 10,02 \mathrm{a}$ & $16,30 \pm 5,25 \mathrm{ab}$ & $15,55 \pm 5,24 \mathrm{~b}$ \\
Número de ovos/fêmea/dia & $1,59 \pm 0,13 \mathrm{a}$ & $1,06 \pm 0,10 \mathrm{~b}$ & $1,08 \pm 0,24 \mathrm{~b}$ \\
Número de ninfas/fêmea/dia & $1,13 \pm 0,11 \mathrm{a}$ & $1,00 \pm 0,11 \mathrm{a}$ & $1,03 \pm 0,30 \mathrm{a}$ \\
Viabilidade de ovos (\%) & $71,53 \pm 3,69 \mathrm{a}$ & $70,01 \pm 4,80 \mathrm{a}$ & $69,83 \pm 3,09 \mathrm{a}$ \\
Eficiência de conversão alimentar (ER) & $4,28 \pm 0,92 \mathrm{a}$ & $3,00 \pm 0,43 \mathrm{~b}$ & $2,75 \pm 0,30 \mathrm{~b}$ \\
\hline
\end{tabular}

${ }^{1}$ Médias ( \pm DP) seguidas de mesma letra na mesma linha, não diferem entre si, pelo teste de Tukey $(P>0,05)$. 

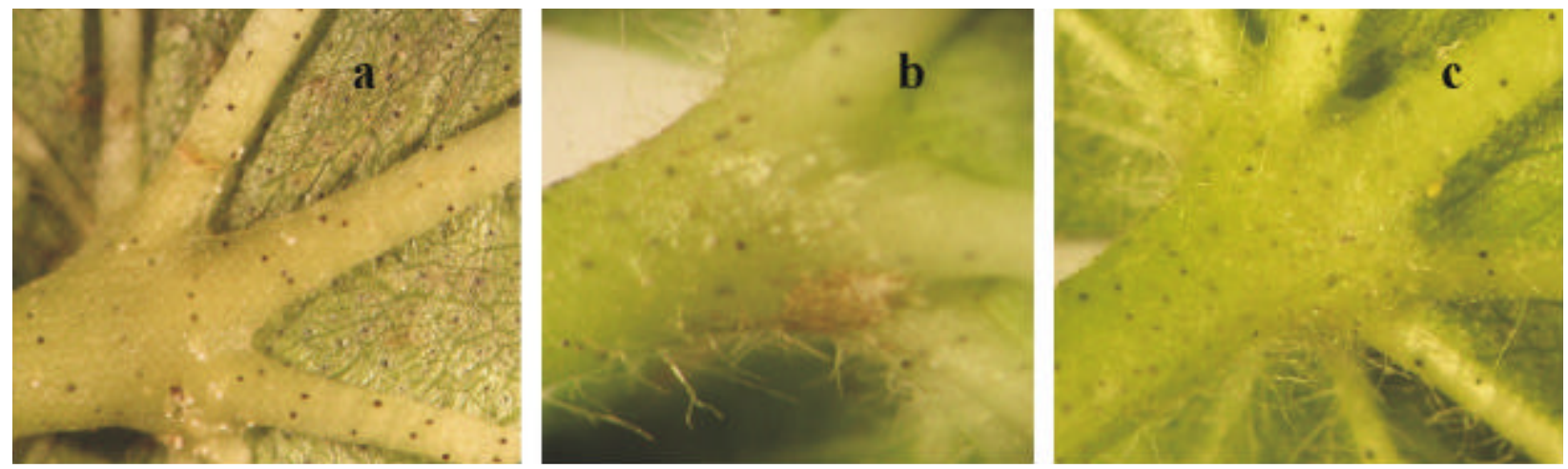

Fig. 2 - Preferência para oviposição das fêmeas de O. insidiosus nos cultivares Antares (a), CNPA7H (b) e Acala 90 (c). Temp. $25 \pm 1^{\circ}$ C; UR $70 \pm 10 \%$; fotofase 14h, Jaboticabal, 2006.

Tabela 4 - Predação total e diária de fêmeas de Orius insidiosus sobre Aphis gossypii em diferentes cultivares de algodoeiro. Temp. $25 \pm 1^{\circ} \mathrm{C}$; UR $70 \pm 10 \%$; fotofase $14 \mathrm{~h}$, Jaboticabal, 2006.

\begin{tabular}{lrr}
\hline Tratamentos & \multicolumn{1}{c}{ Total $^{1}$} & Diária $^{1}$ \\
\hline Antares & $140,15 \pm 69,32^{\mathrm{a}}$ & $5,40 \pm 0,90^{\mathrm{a}}$ \\
CNPA7H & $68,30 \pm 17,67 \mathrm{~b}$ & $3,16 \pm 0,27 \mathrm{~b}$ \\
Acala 90 & $61,09 \pm 20,69 \mathrm{~b}$ & $2,92 \pm 0,49 \mathrm{~b}$ \\
\hline
\end{tabular}

${ }^{1}$ Médias ( $\pm \mathrm{DP}$ ) seguidas de mesma letra, na mesma coluna, não diferem entre si, pelo teste de Tukey $(\mathrm{P}>0,05)$.

Bush et al. (1993) e Mendes et al. (2003) observaram que, predando ninfas de A. gossypii, fêmeas de $O$. insidiosus apresentam longevidade média variando de 9,5 a 9,8 dias, considerada baixa quando comparada com este e outros estudos (TOMMASINI; NICOLI, 1993; Mendes; Bueno, 2001; Tommasini et al., 2004). Segundo Mendes etal. (2003), as fêmeas deO.insidiosus necessitaram de disponibilidade diária de cinquenta ninfas $\left(1^{\circ}, 2^{\circ} \mathrm{e} 3^{\circ}\right.$ estádios $)$ deA.gossypii para produzir diariamente 1,5 ovos, indicandoque presasemínstares iniciais podem comprometer o desenvolvimento do predador, sendocaracterizado pela curta longevidade e baixo número de ovos/fêmea (17,9 ovos em 11,9 dias). Tommasinı et al. (2004) observaram que fêmeas de O. insidiosus ovipositaram de 3,41 a 3,84 ovos/dia, quando alimentadas com ovos de Anagasta kuehniella (Zeller, 1879) e F. accidentalis, respectivamente.

A eficiência de conversão alimentar foi superior quando as fêmeas do predador foram mantidas no cultivar Antares (Tabela 3), havendo necessidade maior de consumo de presas para produção de descendentes. Mendeset al. (2003) observaram que fêmeas de O. insidiosus apresentaram eficiência de conversão alimentar de 4,53 ovos do predador para cada pulgão predado.

Em relaçãoà capacidade predatória totale diária de ninfas de A.gossypii, as fêmeas deO. insidiosusapresentam maior taxa de predação no cultivar Antares, sendo
140,15 ninfas na fase adulta, com média diária de 5,40 ninfas (Tabela 4). Mendesetal.(2003) citaram predação de 7,0 a 7,8 ninfas de $A$. gossypii (de primeiro ao terceiro estádios), quando tinham disponível 10 e 20 indivíduos, respectivamente.

Além do comportamento de busca e predação, observou-se que os adultos do predador O. insidiosus apresentaram comportamento de suç̧ão de seiva e néctar através do nectário extra floral presente nas folhas do algodoeiro, sem lhes causar danos, o que pode indicar que $O$. insidiosus necessita em sua dieta, para se manter nos agroecossistemas, suplementação alimentar extraída de plantas hospedeiras de suas presas, comportamento esse já observado por vários autores (KimAN; Y EARGAN, 1985; Coll, 1996; Coll, 1998). A utilização de substâncias provenientes de plantas tem sido considerada uma alternativa para o percevejo predador em situações de escassez de presas (CRUM et al., 1998; ColL; IzRAYLEVICH, 1997). Este fato é fortalecido em virtude da capacidade dos predadores em obter a umidade de plantas necessária para assegurar sua sobrevivência e reprodução (COHEN, 1982; CoLL; IZRAYLEVICH, 1997). Além disso, especula-se que a obtenção de nutrientes da seiva das plantas pode ser um complemento da dieta, especialmente hormônios e sais minerais, quando as presas utilizadas não suplementam a exigência nutricional (Oliveira et al., 2002).

\section{CONCLUSÕES}

1. A taxa de predação diária de A. gossypii por ninfas de $O$. insidiosus no primeiro, segundo equarto estádios ninfais é afetada negativamente pelos cultivares Acala 90 e CNPA7H.

2. A duração ninfal de $O$. insidiosus não é afetada pelos cultivares de algodoeiro testados.

3. Fêmeas de O.insidiosus ovipositam mais quando confinadas em plantas dealgodoeiro cultivar Antares e menos no cultivar Acala 90. 
4. A taxa de predação diária e total de A. gossypii por fêmeas de $O$. insidiosus é afetada negativamente nos cultivares Acala 90 e CNPA7H.

5. A eficiência de conversão alimentar é mais alta quando as fêmeas de $O$. insidiosus são confinadas no cultivar Antares.

\section{AGRADECIMENTOS}

À FAPESP - Fundação de Amparo à Pesquisa do Estado de São Paulo, pelo suporte financeiro e bolsa concedida a José Eudes de Morais Oliveira (Proc. no. 03/11924-7), ao Prof. Dr.José Carlos Barbosa (FCAV/ UNESP), pelo auxílio na elaboração do delineamento experimental e análise dos dados.

\section{REFERÊNCIAS}

RIOSO, P.S.T. Doenças causadas por vírus em pimentão. Informe Agropecuário, v.18, n.184, p.74-80, 1996.

BUENO, V.H.P. Desenvolvimento e multiplicação de percevejos predadores do gênero Orius Wolff. In: (Ed.). Controle biológico de pragas: produção massal e controle de qualidade. Lavras: UFLA, 2000. p.69-90.

BUSH, L.; KRING, T.J.; RUBSERSON, J.R. Suitability of greenbugs, cotton aphids, and Heliothis virescens eggs for the development and reproduction of Orius insidiosus. Entomologia Experimentalis et Applicata, v.67, n.2, p.217-222, 1993.

CAUQUIL, J. Récents développements dans la lutte contre la maladie bleue du cotonnier en Afrique Centrale. Coton et Fibres Tropicales, v.36, n.2, p.297-304, 1981.

COHEN, A.C. Biochemical and morphological dynamics and predatory feeding habitats in terrestrial heteroptera. In: COLL, M.; RUBERSON, J.R. (Ed.). Predatory Heteroptera: their ecology and use in biological control. Lanham: Thomas Say, 1998. p.21-32.

COHEN, A.C. Water and temperature relations of two hemipteran members of a predator-prey complex. Environmental Entomology, v.11, n.3, p.715-719, 1982.

COLL, M. Feeding and ovipositing on plants by an omnivorous insect predator. Oecologia, v.105, n.2, p.214220, 1996.

COLL, M. Living and feeding on plants in predatory heteroptera. In: COLL, M.; RUBERSON J.R. (Ed.). Predatory Heteroptera: their ecology and use in biological control. Lanham: Thomas Say, 1998. p.89129.
COLL, M.; IZRAYLEVICH, S. When predator also feed plants: Effect of competition and plant quality on omnivore-prey population dynamics. Annals of the Entomological Society of America, v.90, n.2, p.155-161, 1997.

CRUM, D.A.; WEISER, L.A.; STAMP, N.E. Effects of prey scarcity and plant material as a dietary supplement on an insect predator. Oikos, v.83, n.4, p.549-557, 1998.

DE BORTOLI, S.A.; OLIVEIRA, J.E.M. Estudos com pulgão vetor de vírus. Informativo do Manejo Ecológico de Pragas, n.42, p.492, 2005.

DENÉCHÈRE, M. Note sur la distribution et l'évaluation des populations d'Aphis gossypii Glov. (Hymenoptère, Aphididae) sur cotonniers en République Centrafricaine. Cotton et Fibres Tropicales, v.36, n.2, p.271-280, 1981.

HOLLING, C.S. Principles of insect predation. Annual Review of Entomology, v.6, p.163-182, 1961.

ISENHOUR, D.J.; YEARGAN, K.V. Effect of crop phenology on Orius insidiosus populations on stripcropped soybean and corn. Journal of the Georgia Entomological Society, v.16, n.3, p.310-322, 1981.

KIMAN, Z.B.; YEARGAN, K.V. Development and reproduction of the predator Orius insidiosus (Hemiptera: Anthocoridae) reared on diets of selected plant material and arthropod prey. Annals of the Entomological Society of America, v.78, n.4, p.464-467, 1985.

LATTIN, J.D. Bionomics of the Anthocoridae. Annual Review of Entomology, v.44, p.207-231, 1999.

LATTIN, J.D. Economic importance of minute pirate bugs (Anthocoridae). In SCHOEFER, C.W.S.; PANIZZI A.R. (Ed.). Heteroptera of economic importance. Florida: CRC Press, 2000. p.607-637.

LUTTRELL, R.G.; FITT, G.P.; RAMALHO, F.S.;

SUGONYAEV, E.S. Cotton pest management: Part 1. A worldwide perspective. Annual Review of Entomology, v.39, p.517-526, 1994.

MCCAFFREY, J.P.; HORSBURGH, R.L. Biology of Orius insidiosus (Heteroptera: Anthocoridae): a predator in Virginia apple orchards. Environmental Entomology, v.15, n.4, p.984-988, 1986.

MENDES, S.M.; BUENO, V.H.P.; CARVALHO, L.M.; SILVEIRA, L.C.P. Efeito da densidade de ninfas de Aphis gossypii Glover, 1877 (Hemiptera, Aphididae) no consumo alimentar e aspectos biológicos de Orius insidiosus (Say, 1832) (Hemiptera,

Anthocoridae). Revista Brasileira de Entomologia, v.47, n.1, p.19-24, 2003. 
MENDES, S.M.; BUENO, V.H.P. Biologia de Orius insidiosus (Say) (Hemiptera: Anthocoridae) alimentado com Caliothrips phaseoli (Hood) (Thysanoptera: Thripidae). Neotropical Entomology, v.30, n.3, p.423-428, 2001.

OLIVEIRA J.E.M.; DE BORTOLI, S.A.; SANTOS, R.F. Metodologia de criação do pulgão-do-algodoeiro Aphis gossypii Glover, 1877 (Hemiptera: Aphididae) em laboratório. Arquivos do Instituto Biológico, São Paulo, v.72, p.49, 2005. Suplemento 2. Trabalho apresentado na REUNIÃO ANUAL DO INSTITUTO BIOLÓGICO, 18., 2005, São Paulo, SP. Resumos. São Paulo: 2005. Resumo 062

OLIVEIRA, J.E.M.; TORRES, J.B.; CARRANOMOREIRA, A.F.; BARROS, R. Efeito das plantas de algodoeiro e do tomateiro, como complemento alimentar, no desenvolvimento e na reprodução do predador Podisus nigrispinus (Dallas) (Heteroptera: Pentatomidae). Neotropical Entomology, v.31, n.1, p.101108, 2002.

PEÑA-MARTINEZ, R. Identificacion de afidos de importancia agricola. In: URIAS-M, C.; RODRÍGUEZM, R.; ALEJANDRE-A, T. (Ed.). Afidos como vectores de virus en México. Montecillo: Centro de Fitopatologia, Montecillo, 1992. v.2, 135p.

RICHARDS, P.C.; SCHMIDT, J. The effect of selected dietary supplements on survival and reproduction of Orius insidiosus (Say) (Hemiptera: Anthocoridae). Canadian Entomologist, v.128, n.1, p.171-176, 1996a.
RICHARDS, P.C.; SCHMIDT, J. The suitable of some natural and artificial substrates as oviposition sites for the flower bug, Orius insidiosus. Entomologia Experimentalis et Applicata, v.80, p.325-333, 1996b.

SCHMIDT, J.M.; RICHARDS, P.C.; NADEL, H.; FERGUNSON, G.A. A rearing method for the production of large numbers of the insidiosus flower bug, Orius insidiosus (Say) (Hemiptera: Anthocoridae). Canadian Entomologist, v.127, n.3, p.445-447, 1995.

SILVEIRA, L.C.P.; BUENO, V.H. Orius insidiosus (Say, 1832) (Heteroptera: Anthocoridae): sensibilidade ao fotoperíodo e diapausa reprodutiva? Revista Brasileira de Entomologia, v.47, n.4, p.631-635, 2003.

SILVEIRA, L.C.P.; BUENO, V.H.; PIERRE, L.S.R.; MENDES, S.M. Plantas cultivadas e invasoras como habitat para predadores do gênero Orius (Wolff) (Heteroptera: Anthocoridae). Bragantia, v.62, n.2, p.261-265, 2003.

TOMMASINI, M.G.; NICOLI, G. Adult activity of four Orius species reared on two preys. IOBCWPRS Bulletin, v.16, p.281-284, 1993.

TOMMASINI, M.G.; VAN LENTEREN, J.C.; BURGIO, G. Biological traits and predation capacity of four Orius species on two prey species. Bulletin of Insectology, v.57, n.2, p.79-93, 2004.

Recebido em 24/5/06

Aceito em 13/3/08 